\section{OWE-014 WHOLE TRANSCRIPTOME SHOTGUN SEQUENCING REVEALS SIGNIFICANT UPREGULATION OF COLONIC MUCOSAL IMMUNE-MEDIATED ANTI-MICROBIAL MECHANISMS IN PSC-UC}

\begin{abstract}
1,2,3 Mohammed Quraishi* ${ }^{*}$ 1,2 Andrew Beggs, ${ }^{4}$ Animesh Acharjee, ${ }^{1}$ Richard Horniblow, ${ }^{5}$ Amanda Rossiter, ${ }^{1}$ Chris Tselepis, ${ }^{2,3,4}$ Subrata Ghosh, ${ }^{4}$ Georgios Gkoutus, ${ }^{3}$ David Withers, ${ }^{5}$ Willem van Schaik, 2,3,4 Gideon Hirschfield, 1,2,3,4 Tariq Iqbal. ${ }^{1}$ Cancer Genomics and Sciences, University of Birmingham, Birmingham, UK; ${ }^{2}$ University Hospital Birmingham, Birmingham, UK; ${ }^{3}$ Institute of Immunology and Immunotherapy, University of Birmingham, Birmingham, UK; ${ }^{4}$ Institute of Translational Medicine, Birmingham, UK; ${ }^{5}$ Institute of Microbiology and Infection, University of Birmingham, Birmingham, UK
\end{abstract}

\subsection{6/gutjnl-2018-BSGAbstracts.207}

Introduction Primary sclerosing cholangitis is a hepatobiliary disorder of unknown aetiology that is commonly associated with ulcerative colitis (PSC-UC). We have previously demonstrated that patients with PSC-UC have significant alterations in their gut microbiota compared to patients with UC alone and healthy controls. The mechanisms by which these alterations influence pathogenesis is unclear.

Methods Shotgun sequencing was performed on whole transcriptomic libraries of colon biopsies obtained from 10 patients with PSC-UC and 10 healthy controls. Differential gene expression analysis with false discovery rate correction was performed with limma and edgeR. In addition lamina propria mononuclear cells were analysed by multicolour flow cytometry.

Results The colonic transcriptome of patients with PSC-UC demonstrated 6774 significantly differentially expressed genes compared to healthy controls. Gene expression in PSC-UC clustered separate to healthy controls on multidimensional scaling $(p=0.006)$. Gene enrichment analysis revealed 280 significantly altered biological processes. PSCUC was characterised by an overexpression of processes associated with regulation of anti-microbial immune responses compared to healthy controls $(\mathrm{p}<0.0001)$. These immunological responses were enriched with pathways associated with innate, cell-mediated and humoral immunity, autophagy, complement activation and chemokine signalling $(p<0.0001)$. Expression of genes associated with microbial handling including defensins, mannose receptors and antimicrobial peptides were significantly upregulated in PSCUC. Paired CD4 T cell immunophenotyping of colon biopsies revealed a significant increase in the population of Th17 cells and IL-17 producing cells in PSC-UC in comparison to healthy controls $(18.6 \%$ vs $8.5 \% ; \mathrm{p}=0.0007$ $8.5 \%$ vs $5.6 \% ; \mathrm{p}=0.005$ respectively).

Conclusion We have for the first time demonstrated the colonic mucosal transcriptional response in PSC-UC. In comparison to healthy controls, patients with PSC-UC have highly significant expression of genes associated with overrepresentation of multiple immunological processes, many of which are directed against bacteria. Consistently patients with PSC-UC have a significant increase in colonic mucosal Th17 and IL-17 cell population compared to healthy controls.

\section{OWE-015 PROSTAGLANDIN E2 MEDIATES INNATE IMMUNE SUPPRESSION IN ACUTE-ON-CHRONIC LIVER FAILURE VIA THE EP4 RECEPTOR}

Alexander Maini ${ }^{*}$, Natalia Becares Salles, Nathan Robinson, Derek Gilroy, Alastair O’Brien. University College London, London, UK

\subsection{6/gutjnl-2018-BSGAbstracts.208}

Introduction Bacterial infection is a major cause of hospital admission in liver cirrhosis and patients are highly prone to nosocomial infection. Innate immune dysfunction is strongly implicated in these patients.

We showed prostaglandin E2 $\left(\mathrm{PGE}_{2}\right)$ was markedly increased in those patients that demonstrated an immune-suppressive phenotype(1). However, using NSAIDs to reduce $\mathrm{PGE}_{2}$ production is contraindicated due to renal side effects. We aimed to investigate $\mathrm{PGE}_{2}$ downstream signalling pathways to identify a potential druggable target to reverse immune dysfunction in acute-on-chronic liver failure (ACLF).

Methods Peripheral whole blood of healthy volunteers (HV) and ACLF patients was studied. Plasma was analysed for cytokine and $\mathrm{PGE}_{2}$ levels.

Blood was stimulated ex vivo with combinations of lipopolysaccharide (LPS, $1 \mathrm{ng} / \mathrm{ml}), \mathrm{PGE}_{2}(1 \mathrm{ng} / \mathrm{ml})$ and EP2/EP4 receptor blockers for 4 hours. Supernatants were analysed for cytokines. qPCR using Taqman primers was used to assay $\mathrm{PGE}_{2}$ production, enzymes and cell receptors (EP1-4).

Results ACLF patients (mean MELD score 19) were assessed. $\mathrm{PGE}_{2}$ levels in plasma were significantly increased between HV $(163.9 \mathrm{pg} / \mathrm{ml})$ and ACLF $(563.8 \mathrm{pg} / \mathrm{ml})$. ACLF patients showed a marked increase in inflammatory cytokines, including IL-1b, IL-6, IL-8, IL-10 and TNF. Ex vivo stimulation of whole blood with LPS produced significantly lower levels of TNF in ACLF blood $(2.178 \mathrm{ng} / \mathrm{ml})$ vs HV $(6.649 \mathrm{ng} / \mathrm{ml})$. This was matched by IL- 6 production $(12.256 \mathrm{ng} / \mathrm{ml}$ vs $4.492 \mathrm{ng} /$ $\mathrm{ml})$. This was further reduced by addition of exogenous $\mathrm{PGE}_{2}$. Blockade of the EP4 receptor completely reversed reduction in both TNF and IL-6, while EP2 blockade had no effect. qPCR of the EP receptors and $\mathrm{PGE}_{2}$ synthetic enzymes from monocytes and neutrophils from ACLF patients, showed changes in receptor profile and enzymatic machinery used to produce $\mathrm{PGE}_{2}$. These changes were confirmed by Western Blot.

Conclusions ACLF patients are at great risk of morbidity and mortality due to infection caused by multi-factorial immune dysfunction. We have demonstrated that they have a proinflammatory cytokine profile, but an immune-fatigued response to inflammatory stimulus, which mirrors the phenotype seen in the clinic. $\mathrm{PGE}_{2}$ is significantly raised in these patients, however NSAID use is not possible due to risk of renal dysfunction. We demonstrated that $\mathrm{PGE}_{2}$ causes immune dysfunction exclusively via activation of the EP4 receptor and blockade of this receptor restored immune function. EP4 blockade has been shown to have a safe renal profile and we suggest this is a valid target for future immune therapy in ACLF. 


\section{REFERENCE}

1. China $L$ et al. Albumin Counteracts Immune-suppressive Effects of Lipid Mediators in Patients With Advanced Liver Disease. Clin Gastroenterol Hepatol 2017 Aug 28 ;

\section{OWE-016 GENETIC VARIANTS IN PNPLA3 AND TM6SF2 PREDISPOSE TO HEPATOCELLULAR CARCINOMA IN PATIENTS WITH ALCOHOL-RELATED CIRRHOSIS}

\begin{abstract}
${ }^{1}$ Felix Stickel ${ }^{*}$, ${ }^{2}$ Stephan Buch, ${ }^{3}$ Jonas Rosendahl, ${ }^{4}$ Hans-Dieter Nischalke, ${ }^{5}$ Frank Lammert, ${ }^{5}$ Markus Casper, ${ }^{6}$ Arndt Vogel, ${ }^{7}$ Pierre Deltenre, ${ }^{8}$ Florian Eyer, ${ }^{9}$ Daniel Gotthardt, ${ }^{10}$ Thomas Berg, ${ }^{2}$ Jochen Hampe, ${ }^{11}$ Marsha Yvonne Morgan*. ${ }^{1}$ Department of Gastroenterology and Hepatology, University Hospital of Zurich, Switzerland; ${ }^{2}$ Medical Department 1, University Hospital Dresden, Germany; ${ }^{3}$ Department of Gastroenterology, University Hospital Halle/Saale, Germany; ${ }^{4}$ Department of Internal Medicine I, University of Bonn, Germany; ${ }^{5}$ Department of Medicine II, Saarland University Medical Center, Germany; ${ }^{6}$ Department of Gastroenterology, Hepatology and Endocrinology, Hannover Medical School, Germany; ${ }^{7}$ Division of Gastroenterology and Hepatology, University of Lausanne, Switzerland; ${ }^{8}$ Department of Clinical Toxicology, Technische Universität München, Germany; ${ }^{9}$ Department of Internal Medicine IV University Hospital Heidelberg, Germany; ${ }^{10}$ Department of Hepatology, University Hospital Leipzig, Germany; ${ }^{11}$ UCL Institute for Liver and Digestive Health, University College London, UK
\end{abstract}

\subsection{6/gutjnl-2018-BSGAbstracts.209}

Introduction Variants in patatin-like phospholipase domain-containing 3 (PNPLA3; rs738409), transmembrane 6 superfamily member 2 (TM6SF2; rs58542926) and membrane bound $\mathrm{O}$ acyltransferase domain containing 7 (MBOAT7; rs641738) are risk factors for the development of alcohol-related cirrhosis. PNPLA3 rs738409 is also an established risk factor for the development of hepatocellular carcinoma (HCC) within this population. The aim of this study was to explore possible risk associations of TM6SF2 rs58542926 and MBOAT7 rs641738 and the development of HCC.

Methods Risk variants in PNPLA3, TM6SF2 and MBOAT7 were genotyped in 751 cases with alcohol-related cirrhosis and HCC and in 1165 controls with alcohol-related cirrhosis without HCC. Association with the risk of developing HCC was analysed using multivariate logistic regression.

Results The development of HCC was independently associated with PNPLA3 rs738409 (OR 1.84 [95\% CI 1.55-2.18], $\mathrm{p}=1.85 \times 10^{12}$ ) and TM6SF2 rs58542926 (OR 1.66 [1.302.13 ], $\mathrm{p}=5.13 \times 10^{05}$ ) using an additive model and after controlling for sex, age, body mass index and type 2 diabetes mellitus; the risk associated with carriage of MBOAT7 rs641738 (OR 1.04 [0.88-1.24], $\mathrm{p}=0.61$ ) was not significant. The population-attributable fractions were $43.5 \%$ for PNPLA3 rs $738409,11.5 \%$ for TM6SF2 rs58542926, and 49.9\% for carriage of both variants combined.

Conclusions Carriage of TM6SF2 rs58542926 is an additional risk factor for the development of HCC in people with alcohol-related cirrhosis. Carriage of both PNPLA3 rs738409 and TM6SF2 rs58542926 accounts for half of the attributable risk for HCC in this population. Genotyping will allow for more precise HCC risk stratification of patients with alcohol-related cirrhosis, and genotype-guided screening algorithms would optimise patient care.

\section{OWE-017 SURVEILLANCE FOR HEPATOCELLULAR CARCINOMA:} DOES IT IMPROVE OUTCOME?

${ }^{1}$ Mohammad Inamul Haq ${ }^{*}{ }^{2}$ Tee Lin Goh, ${ }^{3}$ Asma Ahmed, ${ }^{1}$ Ewan Forrest, ${ }^{1}$ Ruth Gillespie, ${ }^{1}$ Stephen Barclay, ${ }^{4}$ Matthew Priest, ${ }^{5}$ Jeff Evans, ${ }^{5}$ Janet Graham, ${ }^{6}$ Stuart Ballantyne, ${ }^{1}$ Adrian Stanley. 'Glasgow Royal Infirmary, Glasgow, UK; ${ }^{2}$ University of Glasgow, Glasgow, UK; ${ }^{3}$ Forth Valley Royal Hospital, Glasgow, UK; ${ }^{4}$ Gartnavel General Hospital, Glasgow, UKi ${ }^{5}$ Beatson Oncology Centre, Glasgow, UK; ${ }^{6}$ Queen Elizabeth University Hospital, Glasgow, UK

\subsection{6/gutjnl-2018-BSGAbstracts.210}

Introduction Surveillance for hepatocellular carcinoma (HCC) is recommended by national and international guidelines. However, there are limited data on the impact of surveillance on clinical outcome. Our aim was to compare the stage of disease at diagnosis, treatment employed and survival, among those patients complying with, not complying with, or never entered into, a surveillance programme over a 7 year period at our regional centre.

Methods We analysed data from our prospectively collected regional HCC MDT database on patients diagnosed with HCC from January 2009 to December 2015. Demographics, Child Pugh score and Barcelona-Clinic Liver Cancer (BCLC) stage at diagnosis were collated, as were the treatment strategy employed and survival. We compared the stage of disease, treatment undertaken and survival across 3 pre-identified groups:

1. Compliant with surveillance (enrolled in surveillance and last ultrasound (US) $<9$ months of diagnosis of HCC); 2. Poorly-compliant with surveillance (enrolled in surveillance but last US $>9$ months); 3. Never enrolled into a surveillance programme. Kaplan-Meier, log rank analysis and chi-squared tests were used as appropriate.

Results 641 patients were diagnosed with HCC over this period. Follow up data was available for 638 (99.5\%) patients with mean follow-up 15 months. Mean age at diagnosis was 69 years and $82.3 \%$ were male. HCC diagnoses increased from 62 in 2009, to 143 in 2015. 52.8\% of patients had alcoholic liver diseases, 16.8\% NAFLD and 15.4\% hepatitis C. $160(25.1 \%)$ patients were from Group 1, 54 (8.5\%) from group 2 and $424(66.5 \%)$ from group 3 . There was an increase in the proportion of patients with HCC who were diagnosed in a surveillance programme during the study period $(p<0.01)$. BCLC classification 0/A (very early stage) was seen at diagnosis in $38.8 \%$ patients in group $1,16.7 \%$ in group 2 , and $9.7 \%$ in group $3(\mathrm{p}<0.001)$. Treatment with curative-intent was given to $32.5 \%$ patients in group 1, $14.8 \%$ in group $2 \%$ and $7.8 \%$ in group $3(\mathrm{p}<0.001)$. Mean survival of patients from groups 1,2 and 3 was 32 months, 14 months and 15 months respectively $(p<0.001)$.

Conclusions The annual number of patients diagnosed with HCC at our regional Centre increased by over 100\% during the 7 year study period. A minority of patients diagnosed with HCC were enrolled in a surveillance programme, although the proportion increased over time. HCC Patients enrolled in a surveillance programme had lower BCLC stage at diagnosis, were more likely to receive curative treatment and had improved survival. 